\title{
Developing a thermally compensated electrolyser model coupled with pressurised hydrogen storage for modelling the energy efficiency of hydrogen energy storage systems and identifying their operation performance
} issues.

ALI, D., GAZEY, R. and AKLIL, D. 


\title{
Developing a thermally compensated electrolyser model coupled with pressurised hydrogen storage for modelling the energy efficiency of hydrogen energy storage systems and identifying their operation performance issues.
}

\author{
Dallia Ali ${ }^{\mathrm{a}, \mathrm{n}}$, Ross Gazey ${ }^{\mathrm{b}}$, Daniel Aklil ${ }^{\mathrm{b}}$ \\ a Alexandria University, Alexandria, Egypt \\ b Pure Energy Centre, Shetland, UK \\ n Corresponding author. \\ E-mail address: dolly.ali@hotmail.co.uk (D. Ali).
}

\begin{abstract}
A b s t r a c t
This paper proposes a thermally compensated electrolyser model coupled with a Pressurised Hydrogen Storage model for modelling Renewable Hydrogen Energy Storage Systems needed to support the uptake of renewable energy sources (RES) integration into the grid. The model accurately simulates the output of real world electrolyser and hydrogen storage installations and can be used as a tool for assessing their integrity with intermittent RES.

The model, which has been developed using Matlab/Simulink, incorporates a set of thermally compensated simulation equations to emulate the real world operating installations and define their hydrogen production under different energy configurations (grid connected or directly connected to the RES). It can also be used to determine the efficiency of an electrolyser under cold start or during full temperature operation. The proposed model has proved its ability to emulate real world hydrogen energy systems, and this has been validated by comparing its output to the real-world data logged from an existing field installation.

The developed model has also been used as a tool to assess the performance of an operating real world system, installed in Africa, and which has not been performing as anticipated. Simulation results revealed the presence of a hydrogen leak within this infield installation, and this has been confirmed by an onsite inspection. The developed model has therefore also proved to be able to examine the performance of operating real-world hydrogen energy systems and detect irregularities and operation performance issues.

In summary, this paper proposes a Hydrogen Energy Storage system (HES) model as an enabler simulation tool to support the projected increase of renewable integration into the grid. The model also allows the detection of performance issues within operating HES systems and allows the identification of leaks without the need for conducting intrusive inspections of the installations, hence saving money and time.
\end{abstract}

Keywords:

Energy storage to enable increased renewable integration into the power grid; Green hydrogen generation and energy storage; Modelling the hydrogen systems performance; Hydrogen systems energy efficiency and ROI

\section{Introduction}

Economic and environmental pressures coupled with the significant increase of fossil fuel costs have led to the rapid expansion of electricity generation from Renewable Energy Sources (RES). It has been indicated that $46 \%$ of the global electricity could be delivered from RES by 2050 [1]. Moreover, BPs 2030 energy outlook predicted that Fossil fuel energy source growth is to fall from $83 \%$ to $64 \%$ of market share in favour of renewable energy sources [2].

To enable such an increase in intermittent renewable sources integration into the grid while keeping it stable, the electrical network must have the ability to absorb and store the surplus in the power fed from such fluctuating sources and feed it back when there is a deficit. It has been identified [3] that integrating re-newable hydrogen energy storage systems into the electrical networks allows the absorption of excess energy and the injection of needed energy, hence balancing the network while providing localized services such as green hydrogen fuel for transport or hydrogen injection into the gas grid, or hydrogen for heating. The rationale behind the identification of Hydrogen as an excellent energy storage technology, is that it can act as both a short and long term energy store. With governments around the world moving towards a low carbon economy, it is clear that hydrogen storage will undoubtedly play an important role in facilitating the 'green energy' integration. 
A great challenge that faces the application of Renewable H2 Energy Storage is the ability to accurately determine the hydrogen production of an electrolyser running at the back of a Renewable Energy source. Although a large number of models are available for this purpose, these do not take into account the thermal compensation effect on hydrogen production. This paper therefore proposes a model that can accurately simulate the hydrogen production from an electrolyser fed by a renewable energy source while including the thermal compensation effect.

Other challenges facing the wide application of renewable hydrogen production and storage include the associated high cost and ensuring the system reliable and safe operation. A way forward to cost reduction is lowering the operation and maintenance costs by improving the hydrogen production efficiency and the system performance [4]. To achieve a good efficiency and performance profile, while ensuring secure system operation, the integrity and/or ability of a HES system to handle hydrogen gas securely must be guaranteed and leaks must be quickly identified. If this is not the case, then any leakage will have a direct impact on the overall system efficiency and operating cost as well as a potential safety hazard. The developed thermally compensated electrolyser model can be used in an innovative way to detect $\mathrm{H} 2$ leakages and investigate issues within an operational electrolyser. As $\mathrm{H} 2$ is the lightest and one of the smallest molecules elements in the periodic table and has a buoyant nature, the significant challenge to any hydrogen energy scheme is to ensure that the electrolyser integrity is maintained at all times whilst keeping system availability to end users. The ability to identify possible integrity breaches in a hydrogen energy system can provide an invaluable tool in maintaining operating efficiency and greatly reducing losses, and this is what the proposed novel model allows. The developed model has proven to be able to simply identify a hydrogen leakage within an operating HES system without the need of intrusive inspection. It also allows the quick and easy detection of performance issues within operating HES systems.

This paper is divided into the following seven sections. Section 2 illustrates the need for new electrolyser modelling tools that are specifically developed for HES systems. Section 3 describes the rationale behind the importance of thermally compensated hydrogen electrolyser simulation models. Section 4, illustrates the proposed electrolyser thermal transient algorithm. Section 5 illustrates the development of the proposed model. Section 6 describes the simulation framework and the development of the model in Matlab/ Simulink Environment. Section 7 provides two case studies for validating the developed model. Then the paper ends up with a conclusion in Section 8.

\section{The need for developing new electrolyser modelling algo-rithm tailored to model Renewable hes systems}

Traditionally, electrolysers were mainly switched on and off for producing hydrogen at scheduled times for industrial processes. However, in recent years electrolysers have started to be used in conjunction with renewable energy sources (RES) with the electrolyser either directly connected to the output of the RES or as part of a power grid network balancing mechanism. In contrast to the way large scale industrial electrolysers used to work, these new electrolyser applications (with RES and for grid balancing), are not regularly switched on and off and are operated at variable power levels. In essence, an electrolyser operating in response to renewable generation will often operate in its transitory dynamic states. Thus, in this mode of operation, an electrolyser overall hydrogen output will be affected by its thermal transient characteristics.

In a real world operating electrolyser, the exothermic electrochemical reactions taking place inside the electrolyser will cause it to warm up. The warmer the electrolyser becomes, the more efficient the hydrogen production becomes. Usually electrolysers reach their highest efficiency when operating at their standard operation temperature.

During the period between the electrolyser switch on and reaching its full operating temperature, electrolysis of water occurs at a lower efficiency. In other words, before the electrolyser reaches its full working temperature, it requires more electrical input energy in order to produce hydrogen. This in turn will have an adverse effect on the quantity of hydrogen being produced by the electrolyser.

Throughout the years, different electrolyser modelling tools have been developed and used to examine many aspects of an electrolysis process when coupled with renewable energy [4,5]. The most common purpose of the modelling tools that simulate electrolysis, when combined with renewable energy generators, is to predict their overall hydrogen production output. To this end, a number of models capable of simulating an electrolyser's transient thermal behaviour has been documented [5-7]. These have shown that thermal transients will adversely impact the hydrogen production of an electrolyser. However, the majority of the electrolyser models currently used, within the limited commercially available software tools that offer HES simulation, do not take into account the thermal characteristics of an electrolyser. Further-more, none of these models explored the impact of the transient thermal behaviour of an electrolyser on the overall hydrogen production when connected to RES generation.

In summary, none of the available models and software tools have included the effect of thermal transients on the overall hydrogen production of an electrolyser when connected to renew-able generation or when used in grid balancing applications.

\section{The impact of electrolysers thermal transient effect on their hydrogen production}

Commercially available simulation tools are commonly the ones used for evaluating the performance of an electrolyser within renewable energy storage systems. Among these tools is HOMER which has been developed at the National Renewable Energy Laboratories (NREL) and is considered the most widely used software in modern literature with over 92,000 users worldwide [8]. 
HOMER is probably the most widely recognised and used modelling tool across the academic and industrial community. However, this tool has a significant drawback when simulating hydrogen production from electrolysis in response to renewable generation. In HOMER, the thermal transient effect on the electrolyser' hydrogen production efficiency is not taken into consideration. Instead, HOMER relies on a single assumed value of average production efficiency [9]. This single value assumption disregards the electrolyser' operating temperature and represents an important deviation from the performance of a real operating electrolyser.

Whilst examples of a single assumed value for the electrolyser average hydrogen production can be found in many other commercial software in addition to HOMER, the application of a thermally compensated model is limited to very few modelling tools. Indeed, the only example that has been found in literature in which a thermally compensated model has been applied in a commercially available software for simulating a hydrogen energy storage system is HydroGems [10].

However, HydroGems is only available as part of the Transys 16 simulation environment where the empirical parts of the Hydrogen Energy Storage HES models are calibrated based on the data found in literature and in manufacturer data sheets. It is therefore a significant challenge to calibrate the model parameters effectively within the HydroGems software environment platform. In addition to this, access to the electrolyser source code for calibration within the Transys 16 platform is not available. This therefore restricts its use to the pre-programmed model.

Whilst it would appear that Hydrogems would provide the ideal software platform to perform renewable hydrogen energy storage system simulations, access to the real data from the hydrogen demonstration systems being modelled is essential to ensure correct model operation [7]. In addition, , the work undertaken by the International Energy Association (IEA) in evaluating renewable hydrogen energy projects around the world has revealed a significant drawback, it has been found out that two yearsof analysis were taken on an already operating system in order to calibrate the HydroGems/Transys16 models to work effectively.

To address the HydroGems/Transys 16 model shortcoming while simulating the effects of thermal transients on the electrolyser overall hydrogen production efficiency, a thermally compensated electrolyser model has been developed in this paper. This model has incorporated a set of proposed algorithms to define the overall thermal transience impact on the hydrogen production efficiency when using an electrolyser in conjunction with renewable energy production. The proposed model, as will be shown in the following section, is based on a combination of the heat transfer theory, fundamental thermo-dynamics, and empirical electro-chemical relationships as measured from operating systems.

Fig. 1 demonstrates the impact of heat compensation on the hydrogen production efficiency of the electrolyser. The effect of temperature across the full range of hydrogen production has been simulated using a $30 \mathrm{~kW}$ electrolyser which will be further detailed in the following sections. It can be seen that at lower temperatures the production efficiency is lower than that at full production temperature, $60{ }^{\circ} \mathrm{C}$ in the illustrated example. Therefore disregarding the effects of temperature on the hydrogen production, similar to HOMER, may therefore lead to unrealistic simulation results.

\section{The proposed electrolyser thermal transient algorithm}

To identify the effects of thermal transients on the overall hydrogen production from an electrolyser operated in conjunction with a renewable source, a three step algorithm has been developed. Using the configuration shown in Fig. 2, the first step in the proposed algorithm is carried out and the results are saved. This step involves simulating the hydrogen production from a renewably powered electrolyser, with the electrolyser model being compensated for the effects of temperature on its hydrogen production.

The simulation is then repeated, however this time with the electrolyser temperature being fixed at its full working temperature (i.e. with the effects of thermal transients being ignored) and the results are again saved.

In the final step of the proposed algorithm, the overall effect of thermal transients on the hydrogen production is calculated by subtracting the hydrogen output of the thermally compensated model of step 1 from the hydrogen output of the fixed temperature model of step 2. This yields the cumulative effect of the thermal transients on the overall hydrogen production. The proposed algorithm is shown in Fig. 3.

\section{Electrolyser modelling}

In order to simulate the previous section proposed algorithm, a robust electrolyser model needs first to be developed. This section provides the different formulas used in developing the electrolyser model.

For the past 15 years, electrolyser's model development has been found in literature to relate to direct solar-hydrogen electrolytic demonstration projects. Some other empirical models have also been developed [11-18], but these models have often been unverified or much less detailed. A model that can accurately predict the electrochemical and thermal dynamic behaviour of an advanced alkaline electrolyser has been developed by Øystein Ulleberg [19]. However, for this model to deliver accurate simulation results, three main model components are needed: (a) the voltage/current U-I curve, (b) faraday efficiency, and (c) thermal model. For the electrolyser model proposed within this paper, these components have all been considered and combined together to formulate an accurate and robust electrolyser model. 
Effect of temperature on electrolyser efficinecy

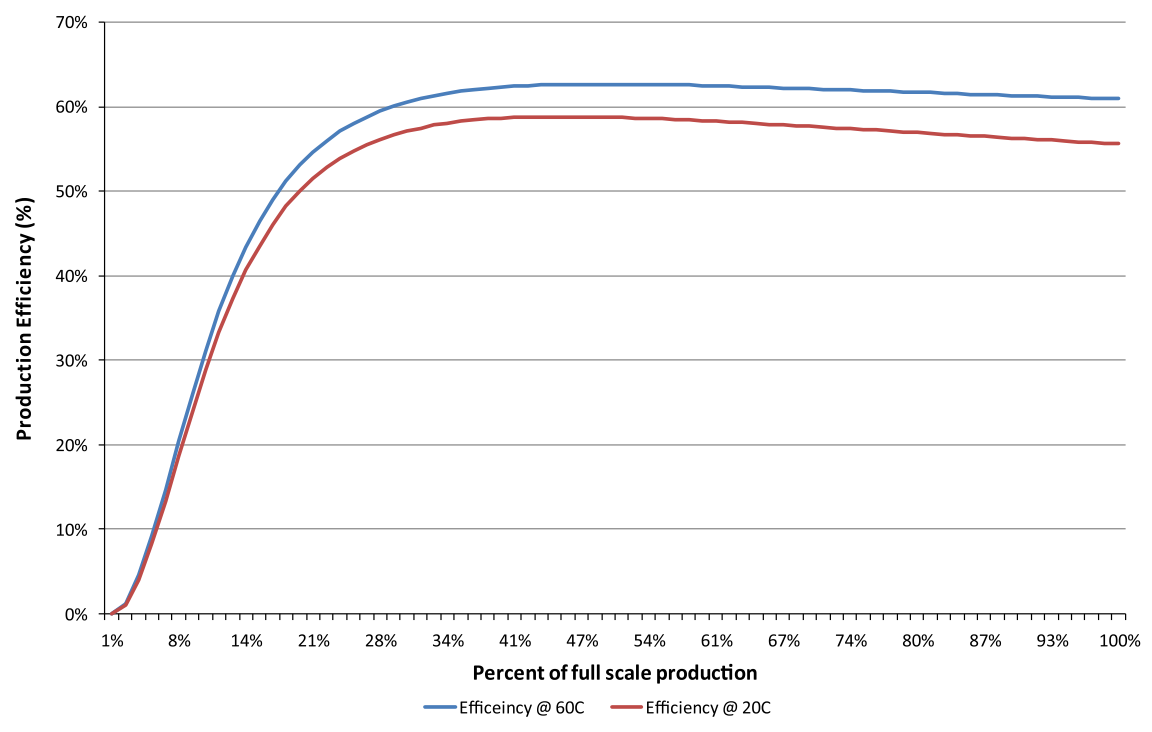

Fig. 1. Impact of hydrogen production on the efficiency of a $30 \mathrm{~kW}$ electrolyser.

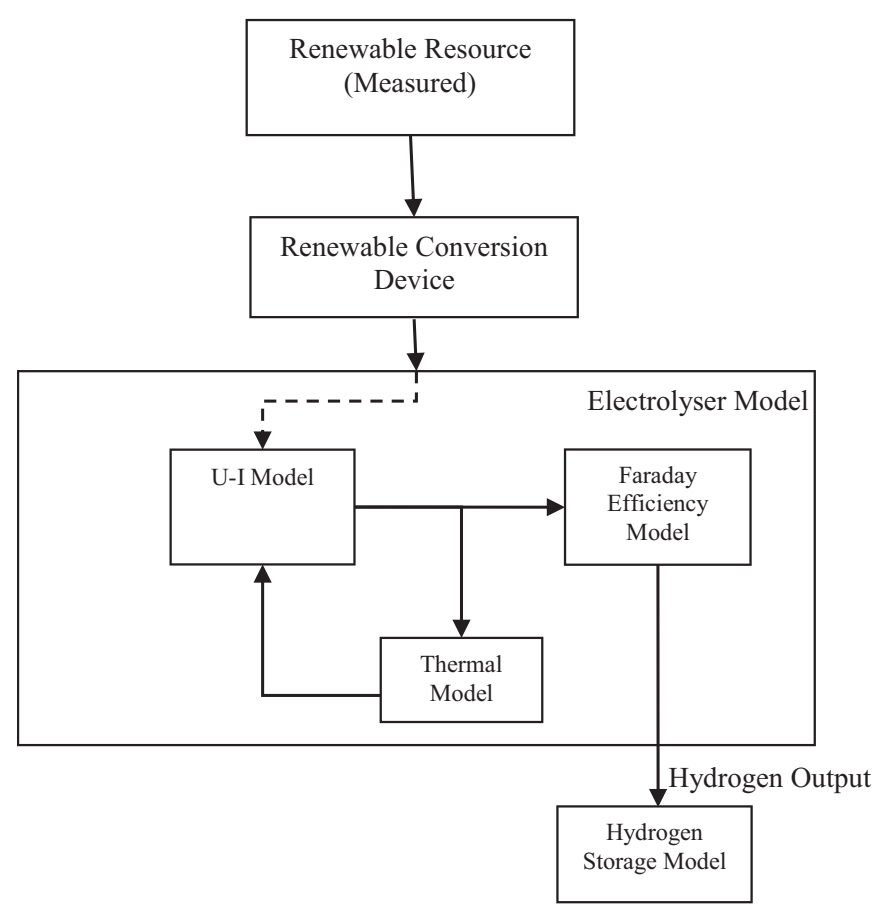

Fig. 2. Electrolyser simulation incorporating the thermal effect on its efficiency. 


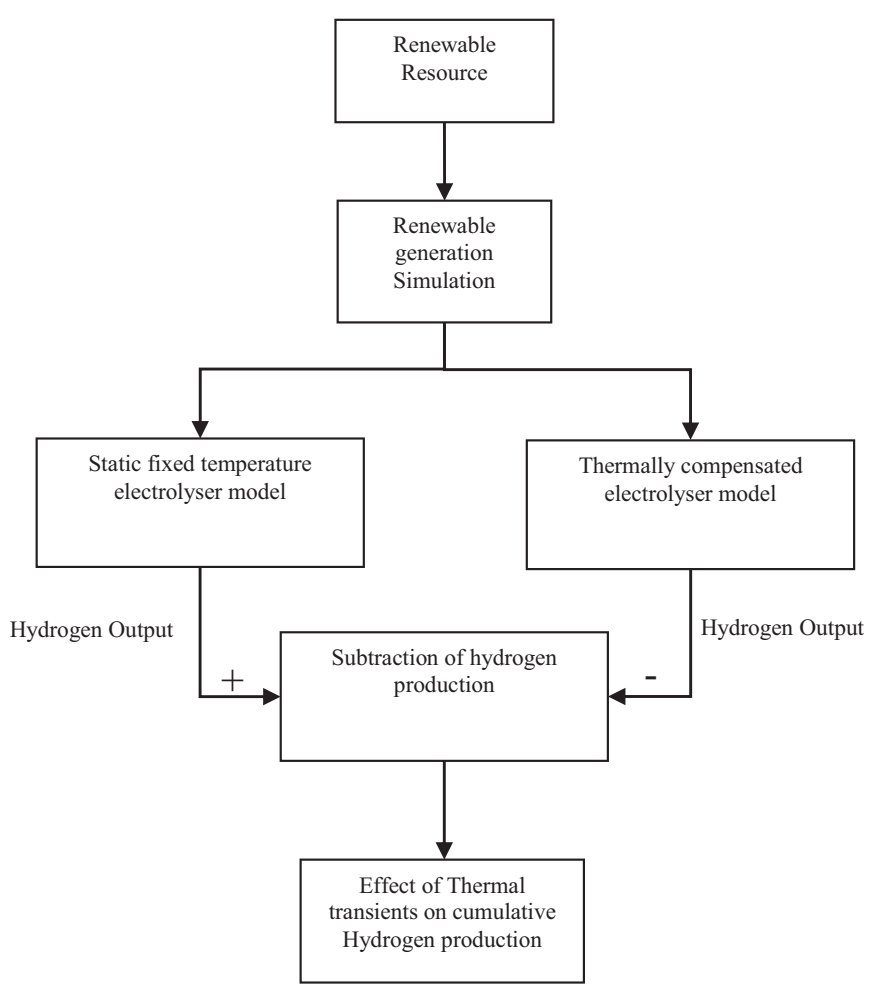

Fig.3. Proposed algorithm to find the impact of thermal transients on the electrolyser hydrogen production.

\subsection{The U-I curve}

An electrolyser operating characteristic is determined by its voltage and current profile. In essence, the quantity of hydrogen produced by an electrolyser varies with the amount of current passing through the electrolytic cell stack.

An electrolyser U-I curve depicts how the electrolytic cell voltage develops as more current is absorbed by the electrolyser to increase the gas output flow. For an ideal electrolyser, this U-I relationship would be a straight line. However, due to the losses that occur in the electrochemistry and cell structure, it becomes a non-linear relationship. This relationship is affected by the ohmic resistance of the electrolyte and electrodes as well as the parasitic loss of 'stray' electrolysis. The parasitic loss of stray electrolysis is a phenomenon where the electrons flow down the electrolyte fluid channels instead of flowing directly between the electrodes themselves.

The voltage $(\mathrm{U})$ required to breakdown the water to produce hydrogen can be expressed in terms of the over-voltage beyond the reversible electrochemical cell voltage (Urev). The voltage required to facilitate electrolytic dissociation of the water molecules is temperature dependant and can be expressed as shown in Eq.(1).

$$
U=U_{\text {rev }}+\frac{\left(r_{1}+r_{2} T\right)}{A} I+s \log \left(\left(\frac{t_{1}+\frac{t_{2}}{T}+\frac{t_{3}}{T^{2}}}{A} I\right)+1\right)
$$

where:

$\mathrm{U}^{1 / 4}$ Voltage (V).

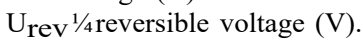

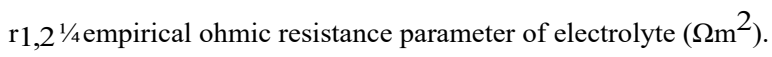

$\mathrm{T}^{1 / 4}$ emperature (K).

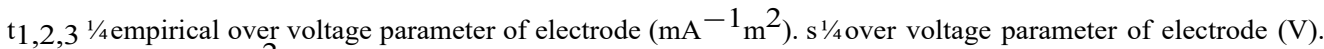

A $1 / 4$ electrode area $\left(\mathrm{m}^{2}\right)$.

I $1 / 4$ current (I).

The reversible cell voltage ( $\mathrm{Urev}_{\mathrm{rev}}$ ) is calculated using the empirical Nernst equation for electrolysis [20] given by Eq. (2). 


\subsection{Faraday efficiency}

The Faraday efficiency is the ratio between the actual and maximum theoretical hydrogen mass that can be produced in an electrolyser. Faraday efficiency losses are caused by parasitic cur-rent losses within the electrolysis cell stack. The parasitic current loss will increase as a percentage of the overall current with decreasing current densities and increasing temperatures. Therefore the percentage of parasitic current loss to the total current flow increases with decreasing current densities.

An empirical equation for the Faraday efficiency is shown in Eq.(3):

$$
\eta_{\mathrm{F}}=\frac{\left(\frac{I}{A}\right)^{2}}{f_{1}+\left(\frac{I}{A}\right)^{2}} f_{2}
$$

where:

$\eta F^{1 / 4}$ Faraday Efficiency.

A $1 / 4$ Electrode area $\left(\mathrm{m}^{2}\right)$.

I $1 / 4$ Current (I).

$\mathrm{f}_{1} 1 / 4$ Faraday efficiency parameter $\left(\mathrm{mA}^{2} \mathrm{~cm}^{-4}\right)$.

$\mathrm{f}_{2} 1 / 4$ Faraday efficiency parameter (number between 0 and 1 ). $\mathrm{f}_{1}$ and $\mathrm{f}_{2}$ are the two constants used in the definition of the

Faraday efficiency of hydrogen production. The first constant $\left(\mathrm{f}_{1}\right)$ is defined in units of $\mathrm{mA}^{2} \mathrm{~cm}^{-4}$ while the second constant used (f2) is defined as an arbitrary number between the values of 0 and 1 . These values are selected empirically.

Faraday's law also models the production rate of hydrogen in an electrolytic cell. The production rate of hydrogen is directly proportional to the transfer rate of electrons at the electrodes. This is equivalent to the electrical current provided by the power supply. Therefore the total hydrogen production rate in an elec-trolysis stack consisting of several cells connected in series can be expressed as shown in Eq. (4):

$$
\dot{n}_{H_{2}}=\eta_{F} \frac{n_{c} I}{z F}
$$

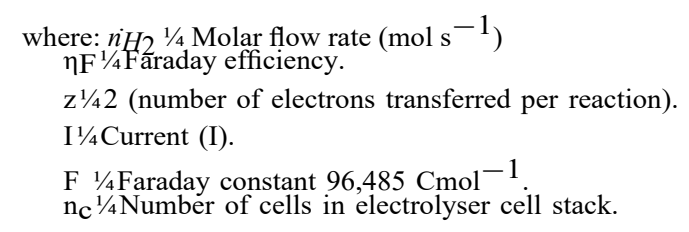

\subsection{Thermal model}

The production of heat in an electrolyser is primarily caused by electrical inefficiencies. The energy efficiency can be calculated from the thermo-neutral voltage $\left(U_{t n}\right)$ and the cell voltage $(U)$ using Eq. (5):

$$
\eta_{e}=\frac{U_{t n}}{U}
$$

where:

$$
\begin{aligned}
& \eta_{\mathrm{e}}^{1 / 4} \text { energy efficiency. } \\
& \mathrm{U}_{\mathrm{tn}}^{1 / 4} \text { thermo neutral voltage } \cong 1.477 \mathrm{~V} \\
& \mathrm{U}^{1 / 4} \text { cell voltage. }
\end{aligned}
$$


As found in literature [21], the value for $U_{t n}$ remains almost constant within the pressure and temperature range considered in this paper (0-1200 $\mathrm{kPa}$ pressure, $0-80{ }^{\circ} \mathrm{C}$ temperature), therefore the value used for $\mathrm{Utn}$ is $1.477 \mathrm{~V}$.

The operating temperature of an electrolyser can be found from the overall thermal energy balance of the electrolysis system. The thermal energy balance of the electrolyser can be expressed as shown in Eq. (6). Eq. (7) calculates the thermal energy created by the electrolysis process, and Eq. (8) is used to calculate the thermal losses of the electrolyser system. Eq. (9) is applied to maintain the electrolyser temperature at or below the maximum temperature specified by the manufacturer. In this case it is always assumed that the electrolyser cooling system is sufficient to remove the excess heat generated by the electrolysis process.

$$
\begin{aligned}
& C_{t} \frac{d T}{d t}=\dot{Q}_{\text {gen }}-\dot{Q}_{\text {loss }}-\quad \text { cool } \\
& \dot{Q}_{\text {gen }}=n_{c}\left(U-U_{t n}\right) I=n_{c} U I\left(1-\eta_{e}\right) \\
& \dot{Q}_{\text {loss }}=\frac{1}{R_{t}}\left(T-T_{a}\right) \\
& \dot{Q}_{\text {cool }}>\dot{Q}_{\text {gen }}-\dot{Q}_{\text {loss }}
\end{aligned}
$$

where:

Q gen $1 / 4$ thermal energy created by electrolysis process.

$\mathrm{Q}$ loss $1 / 4$ thermal energy lost to the environment.

$\mathrm{Q}$ cool $1 / 4$ thermal energy dissipated by cooling system.

$\mathrm{C}_{\mathrm{t}} \mathrm{t}^{1 / 4}$ thermal capacity (or inertia) of electrolyser $\left(\mathrm{JK}^{-1}\right)$.

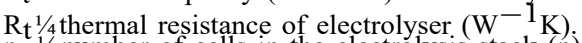

$\mathrm{n}_{\mathrm{c}} 1 / 4$ number of cells in the electrolysis stack (n).

$\eta_{\mathrm{e}} \mathrm{1}^{1 / 4}$ energy efficiency $(\%)$.

$\mathrm{U}_{\mathrm{tn}} 1 / 4$ thermo neutral voltage $(\mathrm{V})$.

$\mathrm{U}^{1 / 4} / 4$ cell voltage $(\mathrm{V})$.

$\mathrm{T} 1 / 4$ electrolyser temperature $(\mathrm{K})$.

$\mathrm{T}_{\mathrm{a}} 1 / 4$ ambient temperature $(\mathrm{K})$.

$t^{1 / 4}$ time.

To calculate the electrolyser temperature as time passes, it is assumed that the electrolyser exhibits a constant heat generation and heat transfer profile for a small time interval of not more than a few seconds. Therefore an intra-time-step steady-state thermal model can be expressed as shown in Eq. (10), where $\mathrm{T}_{\mathrm{ini}}$ is the initial temperature and $\Delta_{\mathrm{t}}$ is the change in time

$$
T=T_{\text {ini }}+\frac{\Delta t}{C_{t}}\left(Q_{\text {gen }}-Q_{\text {loss }}-{ }_{\text {cool }}\right)
$$

\subsection{Pressurised delling}

When hydrogen is produced by the electrolyser, there is a need to store it. Therefore, there is a need to include a hydrogen storage modelling to the proposed model as illustrated in Figs. 2 and 3. This section describes the two main components needed to model pressurised hydrogen storage. The first component is the formula for calculating the pressure, taking into account the gas behaviour. The second component is the $\mathrm{Z}$ factor.The ideal gas relationship can only be used to describe the behaviour of real hydrogen gas accurately at relatively low pressures up to approximately $1450 \mathrm{psig}$ and at normal ambient temperatures. At higher pressures, the ideal gas relationship results become increasingly inaccurate. One of the easiest ways to account for this additional compression is through the addition of a compressibility factor, designated by the symbol $\mathrm{Z}$. The $\mathrm{Z}$ factor is derived from the data obtained through experimentation and it depends on temperature, pressure and on the nature of the gas. The $\mathrm{Z}$ factor is then used as a multiplier to adjust the ideal gas law to fit into the actual gas behaviour as follows: 
where:

$$
\begin{aligned}
& P=\text { absolute pressure in Pascal. } \\
& \rho=\text { density. } \\
& T=\text { absolute temperature in Kelvin. } \\
& \mathrm{R}=\text { universal gas constant }=8.31434 \mathrm{Nm} / \mathrm{mol} \mathrm{K} .
\end{aligned}
$$

\section{Calculating $\mathbf{Z}$}

Research conducted by the National Institute for Standards and Technology developed a mathematical method for calculating compressibility factors using a virial equation based on Pressure $(\mathrm{MPa})$ and Temperature $(\mathrm{K})$. An equation for the density of hydrogen gas that agrees with measurement standards to within $0.01 \%$ from $220 \mathrm{~K}$ to $1000 \mathrm{~K}$ with pressures up to $70 \mathrm{MPa}$, and to within $0.01 \%$ from $255 \mathrm{~K}$ to $1000 \mathrm{~K}$ with pressures to $120 \mathrm{MPa}$, and to within $0.1 \%$ from $200 \mathrm{~K}$ to $1000 \mathrm{~K}$ up to $200 \mathrm{MPa}$ [22], has been developed. Using Eq. (12) and the variable values listed in Table 1, the compressibility factor for hydrogen at different pressures and temperatures can be calculated to a high degree of accuracy.

$$
Z(P, T)=\frac{P}{\rho R T}=1+\sum_{i=1}^{9} a_{i}\left(\frac{100}{T}\right)^{b_{i}}\left(\frac{P}{1}\right)^{c_{i}}
$$

The equation and its constants are defined for pressures in Mega-Pascal (MPa) and temperatures in Kelvin (K). The mass of diatomic hydrogen and the molar gas constant given in Table 1 are obtained from recent publications $[23,24]$. The molar mass is given.

\section{Development of the model using Matlab/Simulink}

The equations that have been presented in the previous section for the proposed model are used in this section to develop a MATLABSimulink model. The developed MATLAB model has then been applied in a novel way to assess the integrity of operating real life hydrogen installations. This section briefly describes the development of the MATLAB model, and the next section will demonstrate its application to an operating Hydrogen installation.

Table 1

Constants used to calculate $\mathrm{Z}$.

\begin{tabular}{llll}
\hline $\mathbf{I}$ & $\mathbf{a}_{\mathbf{i}}$ & $\mathbf{b}_{\mathbf{i}}$ & $\mathbf{c}_{\mathbf{i}}$ \\
\hline 1 & 0.05888460 & 1.325 & 1.0 \\
2 & -0.06136111 & 1.87 & 1.0 \\
3 & -0.002650473 & 2.5 & 2.0 \\
4 & 0.002731125 & 2.8 & 2.0 \\
5 & 0.001802374 & 2.938 & 2.42 \\
6 & -0.001150707 & 3.14 & 2.63 \\
7 & $0.9588528 \times 10^{-4}$ & 3.37 & 3.0 \\
8 & $-0.1109040 \times 10^{-6}$ & 3.75 & 4.0 \\
9 & $0.1264403 \times 10^{-9}$ & 4.0 & 5.0 \\
Molar mass: $\mathrm{M}=2.01588 \mathrm{~g} / \mathrm{mol}$ & & \\
Universal gas constant: $\mathrm{R}=8.314472 \mathrm{~J} /(\mathrm{mol} \mathrm{K})$ & & \\
\hline
\end{tabular}

The hydrogen generation and storage mathematical models described by Eqs. (1)-(12) have been implemented under the Matlab/ Simulink framework to determine the performance of a typical hydrogen energy storage system. Fig. 4 illustrates the in-teraction between the developed Matlab subsystems for the U-I model, the Faraday efficiency model and the thermal model. However, the thermal model shown in the below figure does not take into account any thermal compensation. This is considered a major issue for hydrogen developers as the simulation results will not provide a close enough results to the real practical installations, and this in turn will have negative effects on calculating the real financial return of an installation. As such, the model has been modified to include a newly added thermal compensation factor as will be further detailed in the next subsection. Also note that the pressurised modelling is not shown on the below figure, however it has been taken into account through the H2 Molar Mass. 


\subsection{Compensating the temperature effect in an electrolysis system}

As described above, simulating an electrolyser without considering the thermal compensation leads to a non-negligible error in the simulation which can lead to financial ROI miscalculation. This is especially true during a cold start of an electrolyser. An electrolyser is said to be in a cold start when it is switched on in the following situations: (a) the electrolyser is cold (not heated up and not at its standard operating temperature) and (b) not under pressure. Note that a standard operating temperature for an al-kaline electrolyser is about $80{ }^{\circ} \mathrm{C}$, while for a Proton Exchange Membrane (PEM) electrolyser this is about $70{ }^{\circ} \mathrm{C}$ and for a Solid Oxide Electrolysers (SOE) this vary with the material being used to construct the cells.

In a cold start situation, an electrolyser efficiency is low due to it being cold and not under pressure. In this situation, the electrolyser is required to pressurise itself and heat itself up. This takes a short amount of time if the electrolyser is small. However, the time to pressurise and heat up is dramatically increased as the size of the electrolyser increase. For small electrolysers, this may take one second while for large ones this can take up to several minutes. This is especially true for alkaline electrolysers, while less for the PEM technology. Therefore, the focus here will be on the alkaline electrolysers as they are the ones that suffer the most from the heat compensation effect.

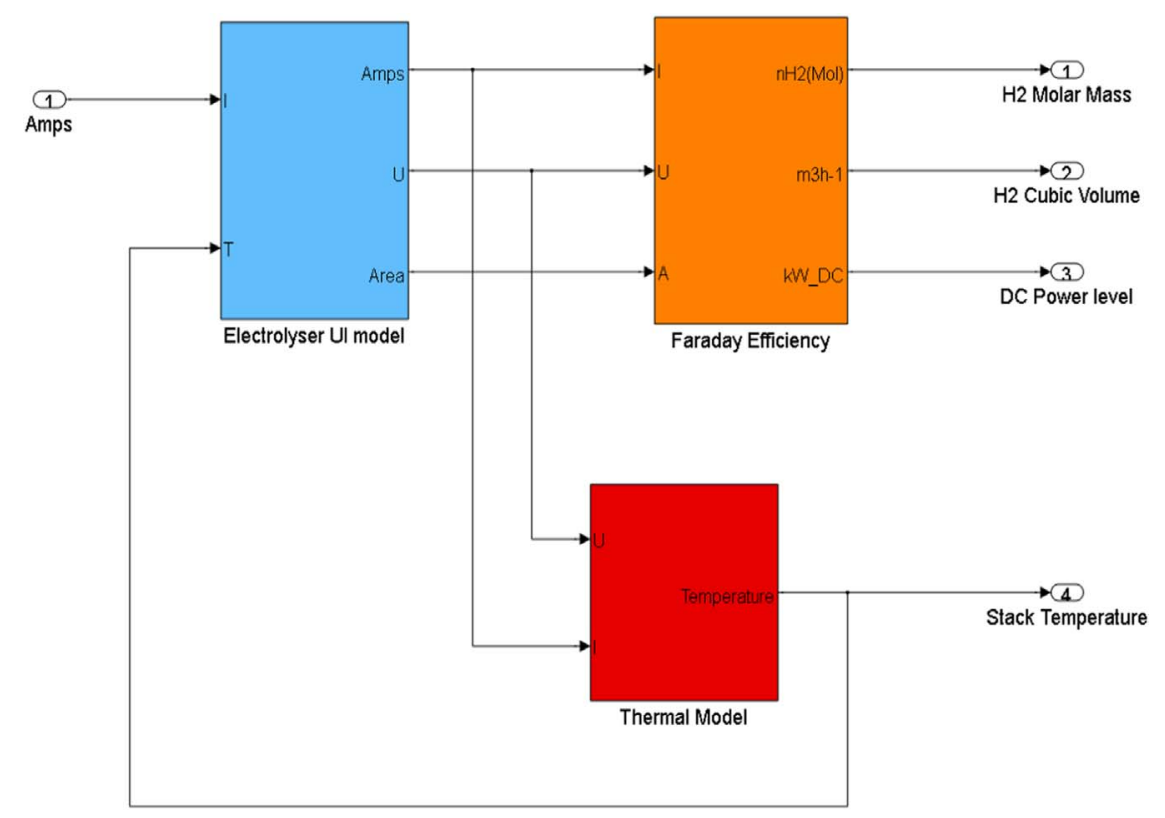

Fig. 4. Interaction between the U-I, the Faraday Efficiency and the thermal subsystem models in Simulink.

To simulate the real world operation of an electrolysis system, it is important to include compensation of the effects of the temperature on the electrolytic process. The exothermic thermal reaction that takes place during electrolysis in an alkaline electrolyser impacts the energy efficiency of the gas generation process and in particular the UI relationships. In other words, as hydrogen is being produced by the electrolyser, an electrochemical reaction takes place at the electrodes. This reaction heats up the electrolyte and the associated electrode materials (this is known as the exothermic reaction). This resulting increase in temperature reduces the cell voltage and cell current needed to generate the hydrogen gas. In other words, the increase in the electrolyser temperature reduces the power requirements of the electrolytic cells, while the hydrogen production is still the same, thereby increasing the efficiency of the system.

Integrating the Thermal model within the proposed model enables the thermal energy efficiency to be calculated, and allows the model to generate output data which are extremely close to the real world electrolyser performance. Fig. 4 depicts the thermally compensated architecture included in the proposed new Simulink model. The new model considers the thermal energy generated by electrolysis (Qgen), the thermal capacity of the electrolyser itself to absorb and dissipate thermal energy (Qloss), and the cooling system to maintain the thermal equilibrium required for an efficient hydrogen generation ( $\left.Q_{c o o l}\right)$. The model utilizes Eq.(10) to calculate the thermal balance of the electrolytic process. Fig. 5 . 


\section{Case studies}

In order to verify the proposed model and validate the concept that thermally compensated electrolyser models are critical for designing and running Hydrogen installations, two case studies are provided in this section. These case studies are carried out on two real-world field installed systems. The first case study is used to verify the developed model. The second case study is used to investigate the operational integrity of an operating electrolyser system and check its performance to identify failures and support the reduction in maintenance requirements.

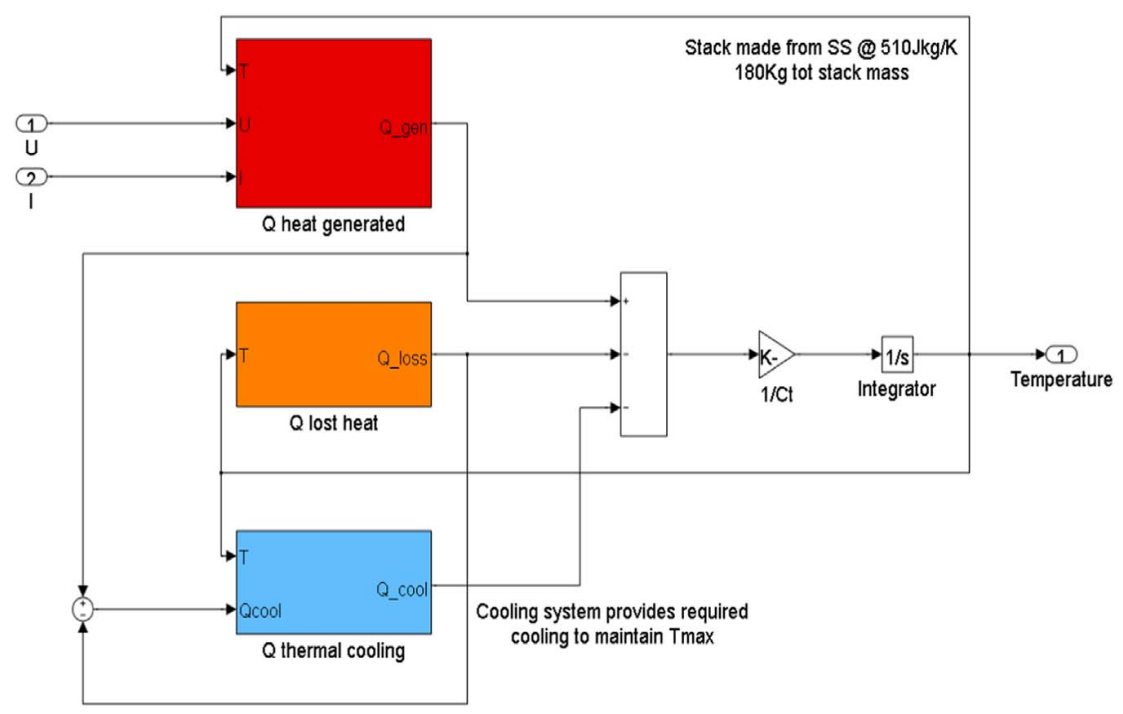

Fig. 5. Thermal compensated model developed under Matlab/Simulink framework.

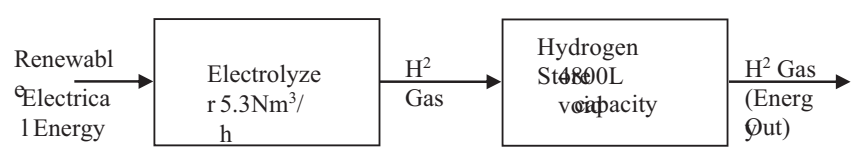

Fig. 6. Hybrid $\mathrm{H}_{2}$ generation and storage model.

Table 2

Table of variables used in electrolyser model.

\begin{tabular}{llll}
\hline Variable & Description & Unit & Value \\
\hline $\mathrm{r}_{1}$ & Electrolyte ohmic resistive parameter & $\Omega \mathrm{m}^{2}$ & 0.0000805 \\
$\mathrm{r}_{2}$ & Electrolyte ohmic resistive parameter & $\Omega \mathrm{m}^{2}$ & -0.0000025 \\
$\mathrm{~A}$ & electrode area & $\mathrm{m}^{2}$ & 0.37 \\
$\mathrm{~S}$ & over voltage parameter of electrode & $\mathrm{V}$ & 0.19 \\
$\mathrm{t}_{1}$ & empirical over voltage parameter of & $\mathrm{A}^{-1} \mathrm{~m}^{2}$ & 1.002 \\
& electrode & & \\
$\mathrm{t}_{2}$ & empirical over voltage parameter of & $\mathrm{A}^{-1} \mathrm{~m}^{2}$ & 8.424 \\
& electrode & & \\
$\mathrm{t}_{3}$ & empirical over voltage parameter of & $\mathrm{A}^{-1} \mathrm{~m}^{2}$ & 247.3 \\
& electrode & & \\
$\mathrm{f}_{1}$ & Faraday efficiency parameter & $\mathrm{mA}^{2} \mathrm{~cm}^{-4}$ & 200 \\
$\mathrm{f}_{2}$ & Faraday efficiency parameter & $0 \ldots .1$ & 0.94 \\
$\mathrm{~V}_{\mathrm{std}}$ & Volume of ideal gas at STP & $\mathrm{m}^{3} \mathrm{~mol}^{-1}$ & 0.0224136 \\
$\mathrm{R}_{\mathrm{t}}$ & Thermal resistance of electrolyser & $\mathrm{W}^{-1} \mathrm{~K}$ & 0.018 \\
$C_{t}$ & Thermal capacity of electrolyser & $\mathrm{JK}^{-1}$ & 300,000 \\
$n_{C}$ & Number of cells in electrolysis stack & $\mathrm{N}$ & 180 \\
$\mathrm{~T}_{\mathrm{a}}$ & Ambient temperature & ${ }^{\circ} \mathrm{C}$ & 20 \\
$\mathrm{~T}_{\mathrm{max}}$ & Maximum operating temperature of & ${ }^{\circ} \mathrm{C}$ & 60 \\
$\mathrm{~T}_{\text {hyst }}$ & electrolyser & & \\
\hline
\end{tabular}




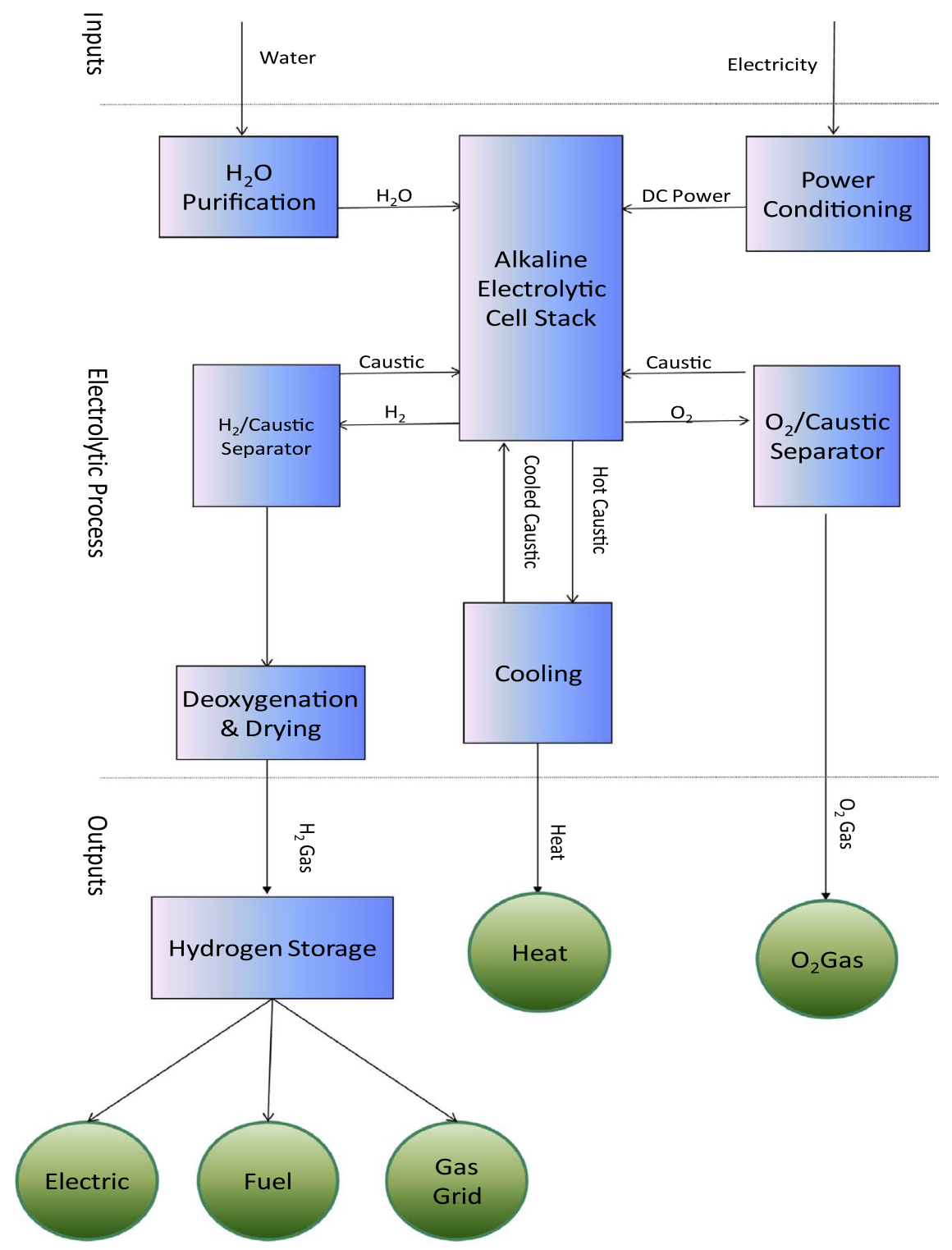

Fig. 7. The developed system model.

Table 3

Table of variables used in storage model.

\begin{tabular}{llll}
\hline Variable & Description & Unit & Value \\
\hline V & Tank volume & $\mathrm{m}^{3}$ & 4.8 \\
Ta & Ambient temperature & ${ }^{\circ} \mathrm{C}$ & 20 \\
\hline
\end{tabular}




\subsection{A.Case study 1 - verification of the developed model using a real world hydrogen system}

A $30 \mathrm{~kW}$ real world alkaline electrolyser, which is operating in an existing field installation, has been chosen here as a case study to verify the developed electrolyser Simulink model. This $30 \mathrm{~kW}$ alkaline electrolyser is capable of developing $5.3 \mathrm{Nm} 3 / \mathrm{h}$ of hydrogen at a pressure up to $1200 \mathrm{KPa}$. It consists of two electrolytic cell stacks, each has 90 cells configured in a series connected array, resulting into 180 cells. The electrolyser is designed to operate at a temperature of $60^{\circ} \mathrm{C}$. As can be seen in Fig. 6, the electrolyser (i.e.the hydrogen generator) is connected to a $4800 \mathrm{~L}$ gas bottle array for the storage of the generated hydrogen at a pressure up to $1200 \mathrm{KPa}$.

The values utilized for the variables of the developed electrolyser model are given in Table 2. While values for the variables of the modular hydrogen storage system, which is connected directly to the electrolysis system, are given in Table 3 . An overview of the overall developed system model is shown in Fig. 7.

A data-log of the current consumed by the real world electrolyser whilst in operation is shown in Fig. 8. The electrolyser temperature and pressure responses to the current are also recorded to be compared to the developed model results as illustrated in Figs. 9 and 10 respectively. Both figures demonstrate that the developed model results are very close to the data collected from the real world system, thus confirming that the proposed model can be used for simulating real world installations accurately.

On further analysing Fig. 9, it can be seen that the electrolyser is switched on at time $133 \mathrm{~s}$ (cold start), and it reaches its operating temperature (and pressure) at time $309 \mathrm{~s}$. The time from the cold start to the operating temperature is therefore 176 s (309-1331/4176 s). It can be concluded that it takes almost three minutes for the electrolyser to reach its operating conditions and substantial amounts of hydrogen will not be produced during this time period. And if the small system takes 3 mins to reach its steady state operation, it can be tangibly assumed that this time will be much higher for a larger size system.

Considering that electrolysers are used to produce hydrogen and that it takes a lot of time for large scale electrolysers to reach their steady states, substantial loss in hydrogen production could be realised. Looking into the financials, it will be consequently affected by these losses in the hydrogen production during the cold start. This means that the inaccurate hydrogen production figures generated using non-thermally compensated hydrogen simulation models will lead to misleading higher financial return figures. Thus, the thermally compensated simulation model developed within this paper will allow the accurate computation of hydrogen production, hence accurately calculating the potential for financial return of a system as close to reality.

\subsection{Case Study 2 - identification of installed systems performance issues}

Having developed the thermally compensated electrolyser model, it was important to further test it on a different application. One of the most unexploited applications of any developed model is its use in a post-installation scenario. When an electrolyser model is developed, it is usually tested in the pre-installation stage to investigate if the system will operate as anticipated when installed in the field. In this section, the developed model is tested in a post-installation scenario to demonstrate that it is further capable to detect an operating electrolyser performance issue before sending the maintenance crew on site.

The developed model has been applied to simulate a hydrogen system that has been operating in Africa for over 4 years. This system has not been performing as anticipated for a couple of weeks and provided a mixture of undesired performance characteristics. The aim of this case study was to determine what was going wrong in a post installation situation. It was suspected that the electrolyser had developed an internal issue and this may need an expensive maintenance visit. Note that the electrolyser involved in this system is identical to that described in Table 2 , and it is connected to a storage system of $2499 \mathrm{~L}$ void volume capacity.

DC current consumed by electrolyser cell stack

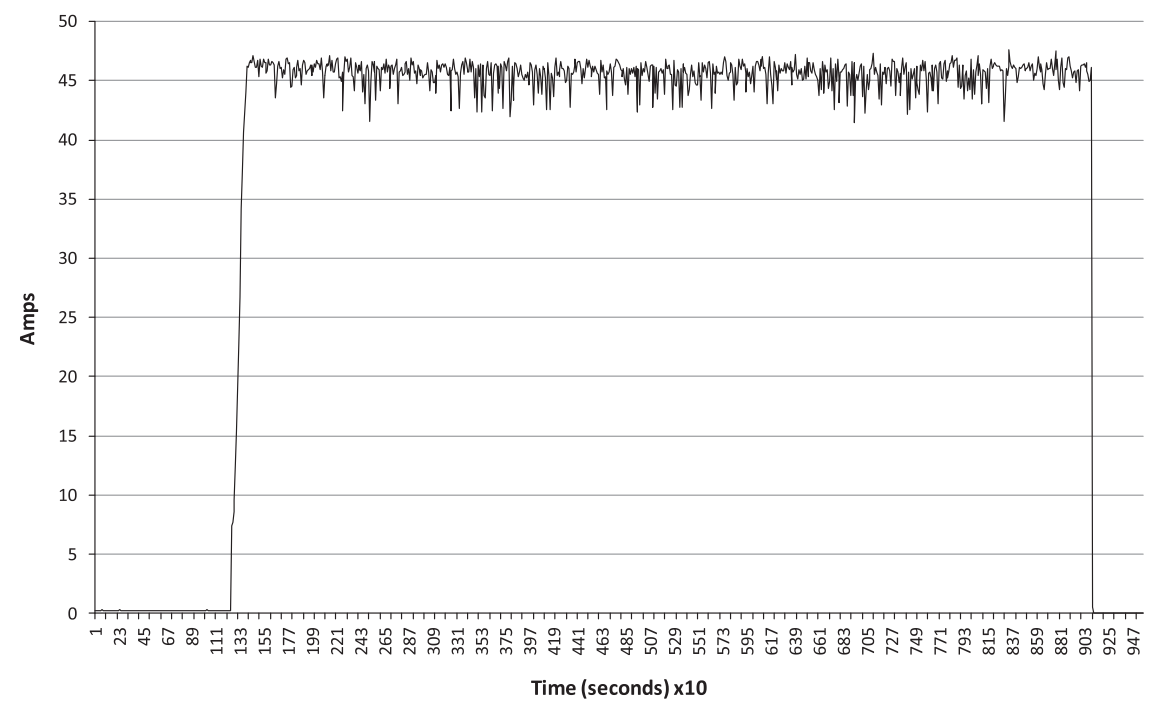

Fig. 8. Electrolyser DC current absorption. 
Temperature (oC)

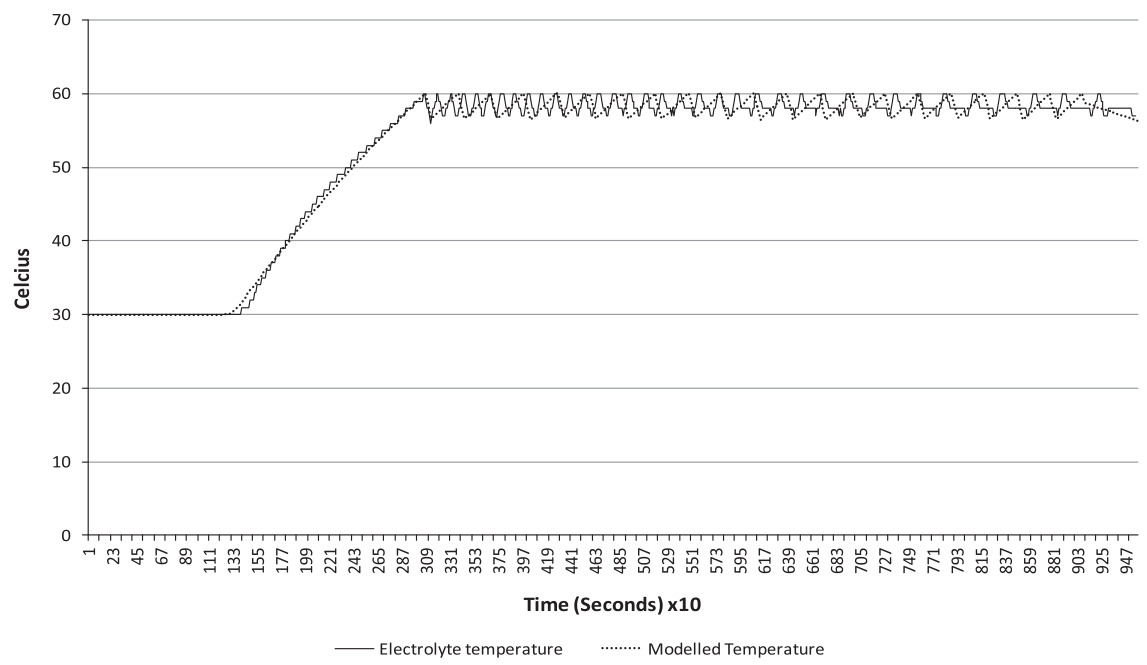

Fig. 9. Electrolyser temperature recorded versus model output.

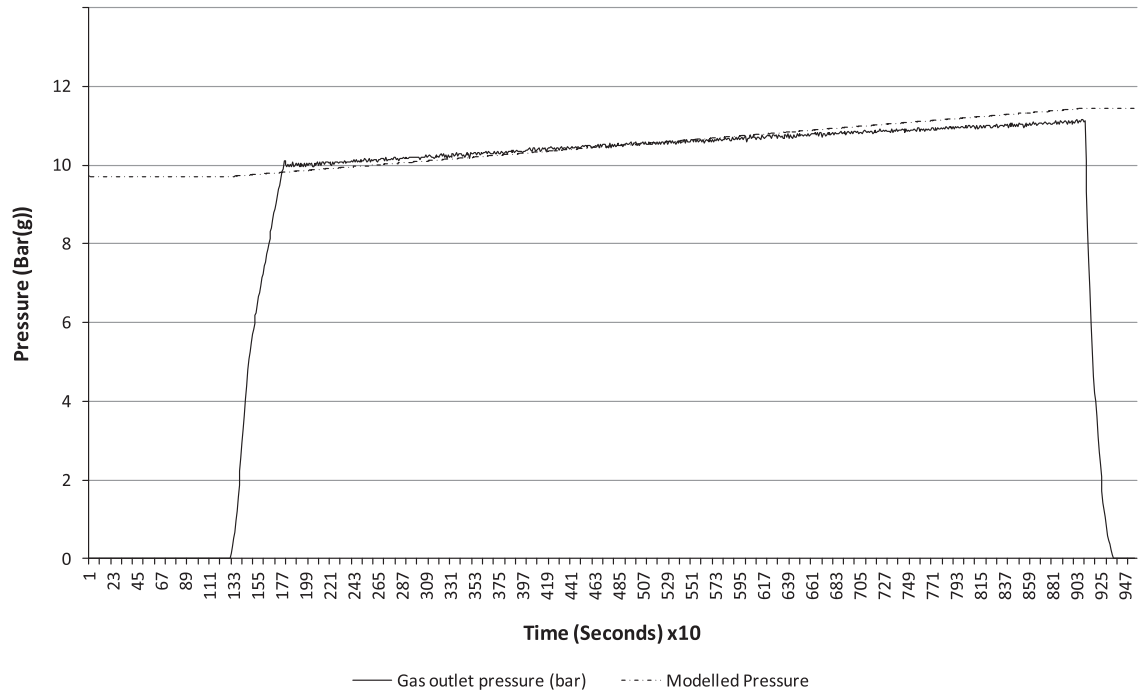

Fig. 10. Storage system pressure recorded versus model output.

When the model results were compared to the system collected data, they suggested two possible performance issues. The first was an early degradation of the stack, however this was disregarded as none of the stacks installed in the field illustrated such a drastic performance issue. The second most probable issue was a hydrogen leak within the installation, and this was confirmed by the clear divergence between the modelled and recorded data. As can be seen in Fig. 10, the model results shows more $\mathrm{H} 2$ production than that of the practical installation, and this in turn suggests a leak within the installed system. Based on the model results, a team of technicians has been dispatched onsite. A detailed inspection of the hydrogen system revealed that a fitting in the pipe that carries the $\mathrm{H} 2$ gas from the electrolyser to the storage system had developed a premature failure. The fast rate of detected leak spray bubbling, shown in Fig. 11, indicates the presence of a leak on two sides of the faulty pipe fitting shown in Fig. 12. This finding has clearly demonstrated the apparent benefit of the proposed model in identifying the source of the problem and saving the time, the maintenance cost, the financial losses through the hydrogen vented in the atmosphere, as well as the safety hazards. 


\section{Case Study Pressure Comparison}

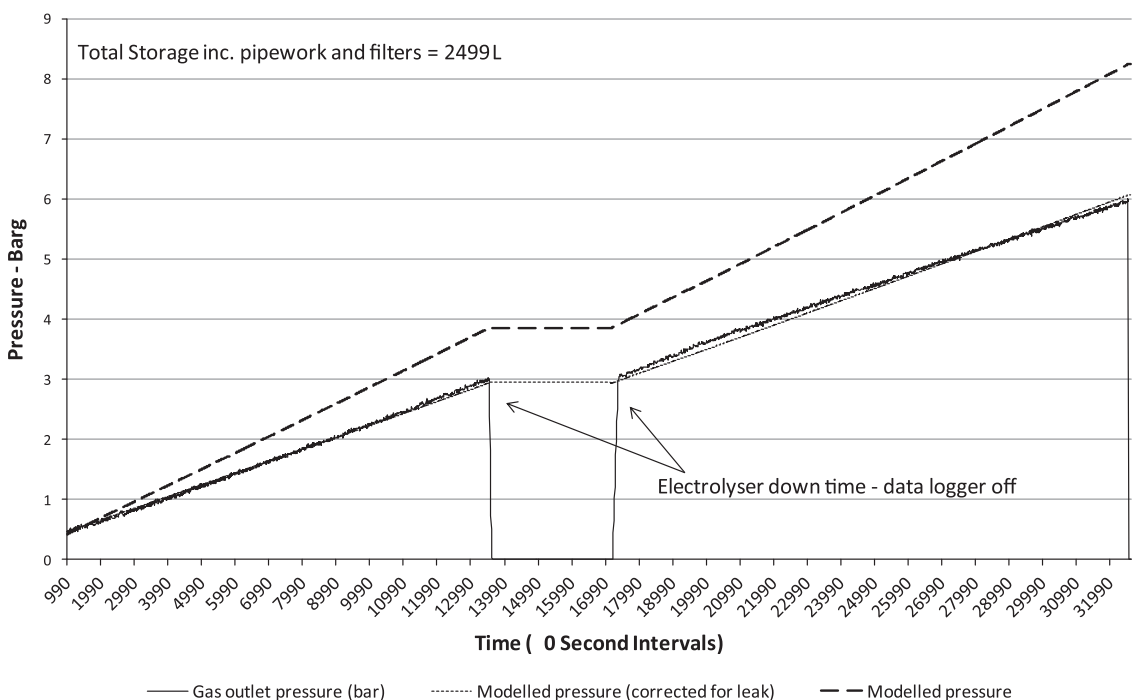

Fig. 11. Divergence of pressures indicating suspected system leak.

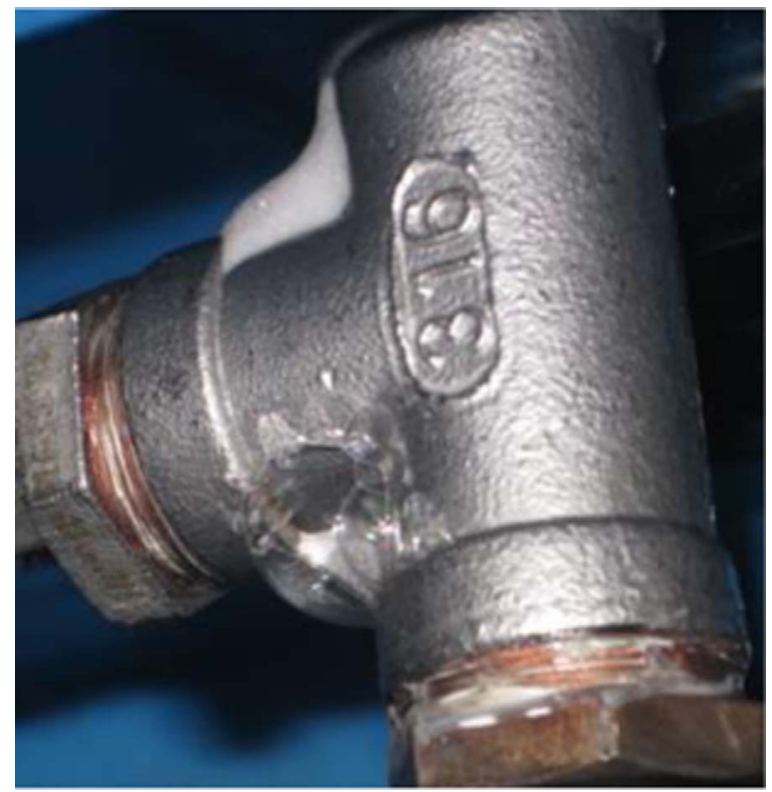

Fig. 12. Source of leakage identified after detailed inspection.

The proposed thermally compensated model has been able to reveal a hydrogen gas leak of about $10.89 \mathrm{~g}$. This loss equated to a $2.3 \%$ reduction in the overall system efficiency. If extrapolated to a large scale system, this could result into a large amount of hydrogen being leaked to atmosphere, costing substantial amount of money to the owner. Moreover, considering the safety of the system, the leak was so small that the leak detection system was not able to detect it. Therefore, in a large scale system with several stacks installed in parallel, many small leaks could occur at different locations without being detected by the safety alarm system and this could lead to financial losses and potentially a hazardous environment. Therefore, utilizing the proposed model couldprovide an early warning of leakages or other issues and thus provide an extra layer of safety and potential for increasing the financial return by developing a predictive maintenance system. 


\section{Conclusion}

This paper presents the development of a thermally compen-sated HES model and demonstrates its innovative application in defining the integrity of operating real world hydrogen installations. The developed model has been verified and validated by comparing its simulation output results to real world operational data.

The developed Matlab/Simulink model has proven to be able to accurately simulate H2 generation and storage systems, which is a key output finding for the hydrogen industry as it allows completion of a real world system performance investigation prior to embarking into expensive capital investment. The developed model has also proved to be useful in simulating operational installed hydrogen systems and assist in the identification of their performance issues accurately.

Modelling the electrolyser with pressurised hydrogen storage has revealed some limitations that must be taken into account in future work. It has been found out that the model developed within this paper is not suitable at high pressures, and therefore future work should consider defining the most appropriate modelling technique at high pressures. Future work should also look into the application of the developed thermally compensated model in quantifying the hydrogen deficit during cold start when using large scale electrolysers, and investigating the associated financial loss and what can be done to reduce or moderate it.

\section{Acknowledgment}

The authors would like to thank those who have made the work described within this paper presentation possible.This research is funded as part of a collaborative research project supported by the Robert Gordon University Research Institute IDEAS, the Environmental Technology Partnership (ETP) and the Pure Energy Centre.

\section{References}

[1] International Energy Agency. Energy Technology Perspectives 2008 - Scenar-ios and Perspectives to 2050. Paris, 2008 , p. 643.

[2] U.S. Energy Information Administration Office of Integrated Analysis and Forecasting. International Energy Outlook 2010. DOE/EIA-0484(2010); July 2010.

[3] Gahleitner Gerda. Hydrogen from renewable electricity: an international re-view of power-to-gas pilot plants for stationary applications. Int J Hydrog Energy 2013:2039-61.

[4] Ursu'a Alfredo, Sanchis Pablo. Static-dynamic modelling of the electrical be-haviour of a commercial advanced alkaline water electrolyser. Int J Hydrog Energy 2012;37(24):18598-614.

[5] Erdinc O, Uzunoglu M. Optimum design of hybrid renewable energy systems: overview of different approaches. Renew Sustain Energy Rev 2012;16(3):1412-25.

[6] Bergen A, Pitt L, Rowe A, Wild P, Djilali N. Transient electrolyser response in a renewable-regenerative energy system. Int J Hydrog Energy 2009;34(1):64-70.

[7] Connolly D, Lund H, Mathiesen BV, Leahy M. A review of computer tools for analysing the integration of renewable energy into various energy systems. Appl Energy 2010;87(4):1059-82.

[8] Software developer statistics published on their home page: 〈http://home renergy.com/〉 [accessed 01.10.13].

[9] Lambert Tom, Gilman Paul, Lilienthal Peter. Micropower System Modeling With Homer. Natl Renew Energy Lab 2005.

[10] Ulleberg Øystein, Nakken Torgeir, Ete Arnaud. The wind/hydrogen demon-stration system at Utsira in Norway: evaluation of system performance using operational data and updated hydrogen energy system modelling tools. Int J Hydrog Energy 2010;35(5):1841-52.

[11] Coll-Mayor Debora, Paget Mia, Lightner Eric. Future intelligent power grids: analysis of the vision in the European Union and the United States. Energy Policy 2007;35(4):2453-65.

[12] Constable Dr John, Moroney Dr Lee. High rewards for wind farms discarding electricity 5-6th April 2011. Renew Energy Found Inf Note 2011.

[13] Hulle Frans Van, Fichaux Nicolas, Sinner Anne-Franziska, Morthorst Poul Erik, Munksgaard Jesper, Ray Sudeshna, et al. Powering Europe: wind energy and the electricity grid. Rep Eur Wind Energy Assoc 2010.

[14] Electricity Networks Strategy Group. Our Electricity Transmission Network: A Vision For 2020. A Summary of an Updated Report to the Electricity Networks Strategy Group, URN 11D/955; February 2012.

[15] Department of Energy and Climate Change. Electricity Market Reform: Con-sultation on proposals for implementation. Presented to Parliament by the Secretary of State for Energy and Climate Change; 2013.

[16] Knab Sebastian, Strunz Professor Kai, Lehmann Dr Heiko. Smart grid: the central nervous system for power supply - new paradigms, new challenges, new services.Berlin: Universität sverlag der TU; 2010.

[17] Manfren Massimiliano, Caputo Paola, Costa Gaia. Paradigm shift in urban en-ergy systems through distributed generation: methods and models. J Appl Energy 2011;88(4):1032-48.

[18] Manfren Massimiliano, Caputo Paola, Costa Gaia. Distributed energy genera-tion and sustainable development. Renew Sustain Energy Rev 2006;10 (6):539-58.

[19] Council of European Energy Regulators (CEER). The Drive towards Smart Grids. European Regulators' Group for Electricity and Gas (ERGEG), FS-10-01; Octo-ber 2010

[20] García-Valverde R, Miguel C, Martínez-Béjar R, Urbina A. Optimized photo-voltaic generator-water electrolyser coupling through a controlled DC-DC converter. Int J Hydrog Energy 2008;33(20):5352-62.

[21] Ulleberg Øystein. Modeling of advanced alkaline electrolyzers: a system si-mulation approach. Int J Hydrog Energy 2003;28(1):21-33.

[22] Waqar A, Qureshi, Nair Nirmal-Kumar C, Farid Mohammad M. Impact of en-ergy storage in buildings on electricity demand side management. Energy Convers Manag 2011;52(5):2110-20.

[23] Moura Pedro S, de Almeida Aníbal T. The role of demand-side management in the grid integration of wind power. Appl Energy 2010;87(8):2581-8.

[24] Knapp Eric D, Samani Raj. Chapter 4 - Privacy concerns with the smart grid, applied cyber security and the smart grid Applied Cyber Security and the Smart Grid. Boston: Syngress,; 2013. p. 87-99 ISBN 9781597499989. 